\title{
Author Correction: Guidance for quantitative confocal microscopy
}

\author{
James Jonkman, Claire M. Brown, Graham D. Wright, Kurt I. Anderson and Alison J. North
}

Correction to: Nature Protocols https://doi.org/10.1038/s41596-020-0307-7, published online 31 March 2020.

The version of the poster initially published online contained several errors. In section 1, at the bottom of the left-hand column, the position of the description "(black dashed lines)" was misleading. The sentence should have said "Fluorescence from outside the focus (black dashed lines), such as from the surface of the sample, is blocked by the pinhole." At the top of the right-hand column in section 2, the word "power" was omitted; the sentence should have read "Use a sensitive microscope to reduce illumination power". In the sentence with the heading "Depth penetration," the units in "50-100 mm" should have been $\mu \mathrm{m}$. In the right-hand figure at the bottom of the column, two lables were transposed: the one for the red line should be "CLSM - fixedcell optimized" and the one for the green line should be "CLSM - live-cell optimized". In the last item in section 3, the units in "10 to $15 \mathrm{~mm}$ " should have been $\mu \mathrm{m}$. In the caption below the image in section 6, "Tubulin labeling yields better resolution with lower wavelength," the word "lower" should have been "shorter". The errors have been corrected in the PDF and print versions of the poster.

Published online: 20 April 2020

https://doi.org/10.1038/s41596-020-0325-5

(C) Springer Nature Limited 2020 